\title{
A FURTHER EXPERIENCE OF THE OPERATION FOR EXCISION OF THE GASSERIAN GANGLION.
}

\author{
BY T. E. GORDON, F.R.C.S.;
}

Surgeon to the Adelaide Hospital, Dublin.

[Read in the Section of Surgery, February 26, 1904.]

Last year, at one of the Surgical Meetings of the Academy, I gave an account of a case of trigeminal neuralgia, for which I performed the operation of excision of the Gasserian ganglion. The removal was not complete, for I left the ophthalmic division and the inner part of the ganglion as well as the sensory root. Up to the present there has, in this case, been no return of the neuralgia.

Some surgeons consider this partial excision sufficient, and, therefore, to be preferred to the complete operation, which is more difficult and dangerous. It is, however, obvious that recurrence of the neuralgia is possible when any part of the ganglion with the sensory root remains intact. Moreover, if one aims at such partial excision one runs a chance of learing behind the entire ganglion, or, at all events, much more of it than that belonging tothe ophthalmic division. In the present case I made a determined effort to remove the ganglion in its entirety, with what measure of success I will narrate when I have given the clinical history.

The patient is a man aged thirty-one years. He was in good health until his present illness began. In June, 1902, he had his first attack of facial neuralgia. The pain had then a darting character-" not like ordinary toothache"-and was referred to his chin and to the front of the left ear. It began one day whilst he was taking his breakfast, continued for about three weeks, and was relieved by the extraction of two teeth. He remained 
free from pain until Christmas of the same year. It is worth noting that the pain continued for two weeks after the extraction of the teeth, and during this time occurred as sudden single darts which caused his head to jerk to the opposite side.

The pain returned on Christmas Day, 1902, but was not very severe till May, 1903; thus, he could sleep and was able to continue his work.

In May it became more severe. In June he had several decayed stumps removed from the left upper jaw. This operation only gave relief for a couple of days. He saw Dr. James Little in October. The pain had continued since May, and would often occur every hour or even every half hour. Eating, speaking, or anything causing movement in his face, might induce an attack. He would sometimes rush away from his work when the pain suddenly seized him. His face would become flushed during the paroxysms, but there was no overflow of tears, nor did the face sweat or become swollen.

He was admitted to the Adelaide Hospital in November, 1903, and remained there till December 20th. During his stay in hospital he was given gr. $\frac{1}{6}$ morphia occasionally, and this was effective. He was also given the usual drugs for the cure of neuralgia, but these only gave temporary relief.

He went home for Christmas, but returned on January lst. The pain was constant whilst at home, and had become more severe than at any time before.

The following is the note taken at the time of his readmission to hospital :--

Since admission on January 1st has had bad pain each night, beginning at about 730 . The pain is "much worse" than whell here before. The act of swallowing appears to cause most pain. The pain is referred to two places in particular-(1) Just in front of the ear above the root of the zygoma ; and (2) the chin, about the mental foramen, and a short distance along the lower jaw. The pain in front of the ear has a boring character. At times he gets a flash of pain along the gum of the upper jaw. He had running from both eyes for a couple of days before coming here. The only tender point is about the lower lip, and this is inconstant. There is no anæsthesia anywhere. 
Such is the history of the case. There are two points to which I draw special attention. The first is the age of the patient; he is much younger than the large majority of cases of trigeminal neuralgia; the second concerns the distribution of the pain; as is usual the third division of the nerve is that principally involved, but there was also, at times, pain in the area of the second division. I do not think there was at any time pain in the branches of the ophthalmic nerve.

Seeing that this patient was, so far from improving under drug treatment, gradually becoming worse, Dr. Little placed him under my care, and I operated on January ith.

As in the last case, so now I performed the Hartley-Krause operation. Last year I gave full details of this method, so that I may now only point out the general features. I turned down a temporal flap and opened the skull with $a \frac{3}{4}$ in. trephine in the upper and back part of the exposed area of bone. This opening I then enlarged to the requisite extent with suitable forceps. Next I retracted the dura as far as the openings in the skull for the passages of the two lower nerve divisions and the middle meningeal artery. All this was accomplished easily and quickly, but the remainder of the operation proved difficult. This difficulty arose from hæmorrhage. This can usually be controlled by plugging the foramina, and tends to stop of itself as a result of the lowered blood-pressure, which is caused by the shock of the operation, but in the present case the bleeding was not thus controllable. I think this was in great part due to the age and vigour of the patient. However, I succeeded eventually in thoroughly opening up Meckel's space and exposing the nerves and ganglion. I also succeeded in evulsing much of the sensory root after dividing at their foramina the 2 nd and 3rd divisions of the nerve. I thought I had removed the whole of the sensory root, but in this I was mistaken, for the patient retains sensation over the entire ophthalmic area.

The patient's recovery is so far complete, and seeing that pain was probably at no time felt in the area of the first division, one may hope that the neuralgia has been permanently cured. For 
some days after the operation there was a partial loss of memory for words, and also a diplopia, which Mr. Swanzy, who kindly examined him for me, found to depend on a loss of power in the inferior rectus.

Now, although this case is so far successful, and may be permanently so, it can scarcely be claimed as a complete success from the operator's standpoint. I intended to. remove the ganglion completely, and I left behind the ophthalmic part of it with its central and peripheral connections.

I have now something to suggest which I think I have learned from this operation and from an operation on the cadaver which $I$ have done since. Let me again insist on the importance of complete excision of the ganglion. The cause of the neuralgia is a neuritis - a neuritis ascending from the periphery - and when the pain extends to the area of other divisions of the nerve such extension is due to a spread of the choice inflammation to this other division by way of the Gasserian ganglion. Now, if these statements are true, and I have the very best authority for them, then it follows that if any part of the ganglion remain inflammatory change may continue and spread to the remaining ophthalmic division. As further argument against partial removal, let me again say that if the operator aims at removing only the outer part of the ganglion he may fail to remove any part of it. This is not imaginary; it has, as I think I said last year, actually happened in operations performed by good surgeons. But, without supposing so extreme a case, if any of the cells remain which belong to the lower divisions of the nerve, from them fibres may grow outwards and with a restoration of function there may be, indeed probably will be, a return of the neuralgia. I have so far spoken of the relation of the ganglion to the nerve divisions; its relation to. the great sensory root is different. 
If the entire sensory root has been successfully torn from its attachment to the pons, nothing is more improbable than that the ganglion cells should ever again establish a central connection. It seems, then, to me that the chief aim which the surgeon should have before him in performing this operation is the isolation and complete evulsion of the sensory root. You will remember that in the operation, as usually performed, the space of Meckel is opened by splitting the dura between the foramen rotundum and foramen ovale, and the dissection of the ganglion is carried out mainly from before backwards. Thus it happens that the operator reaches the sensory root as a final step in the operation, and when his mind is much fatigued if, as in this case of mine, the dissection has been difficult owing to hæmorrhage. My suggestion is that Meckel's space should be opened from the foramen ovale in a backward instead of a forward direction. The middle meningeal artery must, of course, be divided, but this is a matter of no importance. Having opened the space in which the ganglion lies in this way, I propose, in my next operation, to at once proceed to the identification and separation of the sensory root. If, then, the evulsion is well done I think one might leave both the ganglion and the nerve divisions untouched.

Having put forward this suggestion, the main purpose of my paper is accomplished. The suggestion is briefly this-A complete evulsion of the sensory root will produce the same effect as removal of the ganglion, and the effect will be permanent. This being so, the operator should from the first aim at the isolation and evulsion of this sensory root, and it might simplify the operation to open the space of Meckel behind the foramen ovale and third division of the fifth nerve instead of in front of it. 